\title{
BMJ Open Functional changes in the visual cortex in preoperative and postoperative patients with intermittent exotropia: study protocol for a non-randomised case-control clinical trial
}

\author{
Yanan Guo, ${ }^{1}$ Jing Fu $\left(1,{ }^{1}\right.$ Jie Hong, ${ }^{1}$ Zhaohui Liu, ${ }^{2}$ Xueying $\mathrm{He}^{2}$
}

To cite: Guo Y, Fu J, Hong J, et al. Functional changes in the visual cortex in preoperative and postoperative patients with intermittent exotropia: study protocol for a nonrandomised case-control clinical trial. BMJ Open 2022;12:e055848. doi:10.1136/ bmjopen-2021-055848

- Prepublication history for this paper is available online. To view these files, please visit the journal online (http://dx.doi. org/10.1136/bmjopen-2021055848).

Received 27 July 2021 Accepted 02 February 2022

Check for updates

(C) Author(s) (or their employer(s)) 2022. Re-use permitted under CC BY-NC. No commercial re-use. See rights and permissions. Published by BMJ.

${ }^{1}$ Department of Ophthalmology, Beijing Tongren Hospital, Capital Medical University, Beijing,

China

${ }^{2}$ Department of Radiology,

Beijing Tongren Hospital, Capital Medical University, Beijing,

China

Correspondence to

Dr Jing Fu; fu_jing@126.com

\section{ABSTRACT}

Introduction Intermittent exotropia (IXT) is the most common type of divergent squint. IXT is primarily a cortical neurologic dysfunction disorder, occurring as a result of the insufficient maintenance of sensory and motor fusion. Recent reports have demonstrated the relationship between IXT and visual cortical impairment. We planned to assess blood oxygen level-dependent (BOLD)-functional magnetic resonance imaging (fMRI) in patients with IXT during the preoperative and postoperative follow-ups to evaluate the functional changes in the visual cortex.

Methods and analysis A total of 90 Chinese subjects will be recruited, and their ages will be between 18 and 40 years old. The subjects will include the surgical treatment (ST) group (45 subjects with IXT who will undergo surgery) and the HC group (45 age-matched, sex-matched and education-matched healthy volunteers). The assessments will include the following aspects: fMRI and general ophthalmic examinations, optometry measurements and strabismus-related tests, such as the ocular deviation, binocular vision test and Newcastle Control Score (NCS). Each subject will complete the resting-state BOLD-fMRI, and the sequences will include echo planar imaging (EPI) pulse and 3-dimensional brain volume (3D-BRAV0) to acquire high-resolution images. The follow-up schedule will be 6 and 12 months after the surgery. The primary outcome will be determined by cortex changes in BOLDfMRI in the ST group before and after surgery. We will also compare the $\mathrm{HC}$ group with the preoperative subjects in the ST group. The secondary outcomes will be changes in strabismus-related examinations, such as binocular visual function and NCS.

Ethics and dissemination Ethical approval was obtained from the Medical Ethics Committee of Beijing Tongren Hospital. We plan to publish the results of this study in a peer-reviewed journal article.

Trial registration number ChiCTR2100048852

\section{INTRODUCTION}

Intermittent exotropia (IXT) is the primary type of exotropia, which is a transitional condition between exophoria and constant exotropia. ${ }^{1-3}$ There are plenty of epidemiology articles that reported the prevalence of
Strengths and limitations of this study

- The present study is advanced and aims to explore the functional changes in the visual cortex in patients with intermittent exotropia (IXT) before and after surgery through resting-state 3.0 T blood oxygen level-dependent-functional magnetic resonance imaging. We also investigate the relationships between changes in the cortex and ocular examination.

- The follow-up comparison between the visual cortex and ophthalmic changes will enrich our understanding of the impairment and plasticity of visual function in patients with IXT.

- Loss to follow-up of participants was possible after the surgery in this study.

- The present study focuses on patients with IXT with indications for surgical intervention. We did not study the different subtypes or severities of IXT.

strabismus or exotropia among different countries and ethnic groups, but few articles specifically address the prevalence or incidence of IXT. It is estimated that the prevalence of IXT is between $0.12 \%$ and $3.5 \%$ worldwide among different ages and races, and IXT accounts for over $60 \%$ of exotropia. ${ }^{1-6}$ The prevalence of IXT is $3.24 \%-3.9 \%$ in China, and IXT accounts for $50 \%-90 \%$ of exotropia among all age groups. ${ }^{7-10}$

IXT is frequently noted in infancy or early childhood as an outward deviation of one eye. IXT occurs during the disruption of binocular vision, especially when fusional compensatory mechanisms are compromised. ${ }^{11}$ IXT is predominantly a cortical neurologic dysfunction disorder, although with unclear pathology and etiological mechanisms. ${ }^{12-16}$ Binocular vision impairment frequently occurs with disease progression, such as accommodation and vergence deficiency, defective fusion and a loss of 
stereopsis. Gradually, IXT breaks down into constant exotropia. ${ }^{1611-14}$ In addition to cosmetic consequences, IXT can have a dramatic impact on education, socialisation and vision-related quality of life if not treated in a timely manner. ${ }^{17} 18$ Current treatment strategies include the correction of refractive errors, patching therapy, extraocular muscle surgery, botulinum toxin injection and perceptual training. ${ }^{11}$

The fusion function facilitates the brain to translate disparities in information between the images of each eye into a vergence command to enable generate perception, which is a complex cerebral activity, including motor fusion and sensory fusion. ${ }^{19}$ However, fusion is important for orthophoria. Binocular fusion dysfunction possibly disrupts the coordination and balance of the two visual axes, thus leading to misalignment, which is one of the key causes of strabismus. ${ }^{20}$ Surgery is currently the main treatment to align a deviated or strabismic eye for both functional and cosmetic reasons. Weakening the lateral rectus with or without strengthening the medial rectus is commonly used to restore normal eye position. Surgical intervention can restore central fusion and stereoacuity in some patients with IXT. Distance stereopsis can recover even if surgery is postponed until adolescence. ${ }^{21}$

Recently, functional magnetic resonance imaging (fMRI), especially blood oxygen level-dependent (BOLD)-fMRI, has become an important neuroimaging technique used to locate and quantify microscopic functional neurologic changes, due to the advantages of being well-developed, high-quality, high-resolution, repeatable and non-invasive. ${ }^{22}$ BOLD-fMRI has been used to evaluate visual cortex function for decades. ${ }^{22-24} \mathrm{Li}$ et al found increased activation intensity in the bilateral superior parietal lobule and inferior parietal lobule in subjects with IXT, which indicated that binocular fusion involves a complicated brain network including several regions. These cortical activities compensate for binocular fusion dysfunction in IXT. ${ }^{25}$ In patients with concomitant exotropia, Voxel-Based Morphometry (VBM) and voxelbased analysis of DTI showed structural abnormalities in occipital and parietal areas, especially in the middle occipital gyrus and inferior parietal lobule (supramarginal gyrus), which play important roles in the dorsal visual pathway. This result suggested that the dorsal visual pathway was abnormal or impaired. ${ }^{26}$ In patients with concomitant exotropia, ReHo values were increased in the right inferior temporal cortex/fusiform gyrus/cerebellum anterior lobe, right lingual gyrus and bilateral cingulate gyrus, which may indicate that patients with concomitant exotropia had brain function compensation for fusion function. ${ }^{27}$ In infantile esotropia, Yang et al found increased BOLD signals in the left cingulate gyrus, bilateral precuneus and left angular gyrus. The bilateral frontal gyrus and left lingual visual cortex regulate normal fusion function in human eyes. ${ }^{20}$ In patients with congenital concomitant strabismus, amplitude of low-frequency fluctuation (ALFF) values decreased in the bilateral middle frontal gyrus and increased in the bilateral posterior cerebellar lobe and left angular gyrus, which may reflect the underlying pathologic mechanism of congenital strabismus. ${ }^{28}$ However, the exact cerebral region and activation changes during the progression of IXT remain unclear, and plasticity in the process of treatment needs to be clarified. Since IXT is related to fusion function and stereopsis impairment, using BOLDfMRI examination in follow-ups may provide a reasonable method to further reveal the pathological mechanism of IXT.

\section{METHODS}

The current prospective, non-randomised clinical trial is designed to evaluate the functional changes in the visual cortex through resting-state 3.0 T BOLD-fMRI pre-IXT and post-IXT operation and to compare the changes at each time point during the 1-year follow-up. Other changes are also compared over the study period, including general ophthalmic examinations, optometry examinations, strabismus-specialised examinations, binocular visual function examinations, and NCS.

\section{Study setting and responsibilities}

The clinical trial will be conducted in Beijing Tongren Hospital, a large tertiary centre with specialist ophthalmology clinics and radiology clinics in Beijing, China. The composition of the steering committee will be as follows: JF will be the primary investigator (PI); ZL will be the co-PI; JH will be the sub-PI and YG and XH will be consultants. The steering committee will provide the final approval of the protocol and any changes to the procedure during the clinical trial. $\mathrm{JH}$ will be in charge of supervising the conduction of the study, including staff training and assessment, protocol decisions and amendments, form development, data management, data analyses and quality control. YG and XH will be responsible for data collection and recording.

\section{Study design and recruitment}

This study is designed as a non-randomised case-control clinical trial. Recruitment will officially begin from 1 September 2021. A total of 90 subjects will be recruited, and each of the participants in the ST group will be followed for 1 year.

Potential participants will be recruited for clinical trials in Tongren Hospital through two primary processes: (1) an ophthalmologist referral during daily routine outpatient clinical work and (2) an optometrist referral from optometry clinics during myopia treatment. All of the potentially eligible participants will be contacted by study staff who will explain the process of the study in detail to ensure that patients understand the entire clinical trial. Prior to signing informed consent, detailed information about the study procedure will be provided to participants, including the purpose of the research, examinations, follow-up duration and possible risks. If they are interested, the subjects will be seen in the clinic to sign 


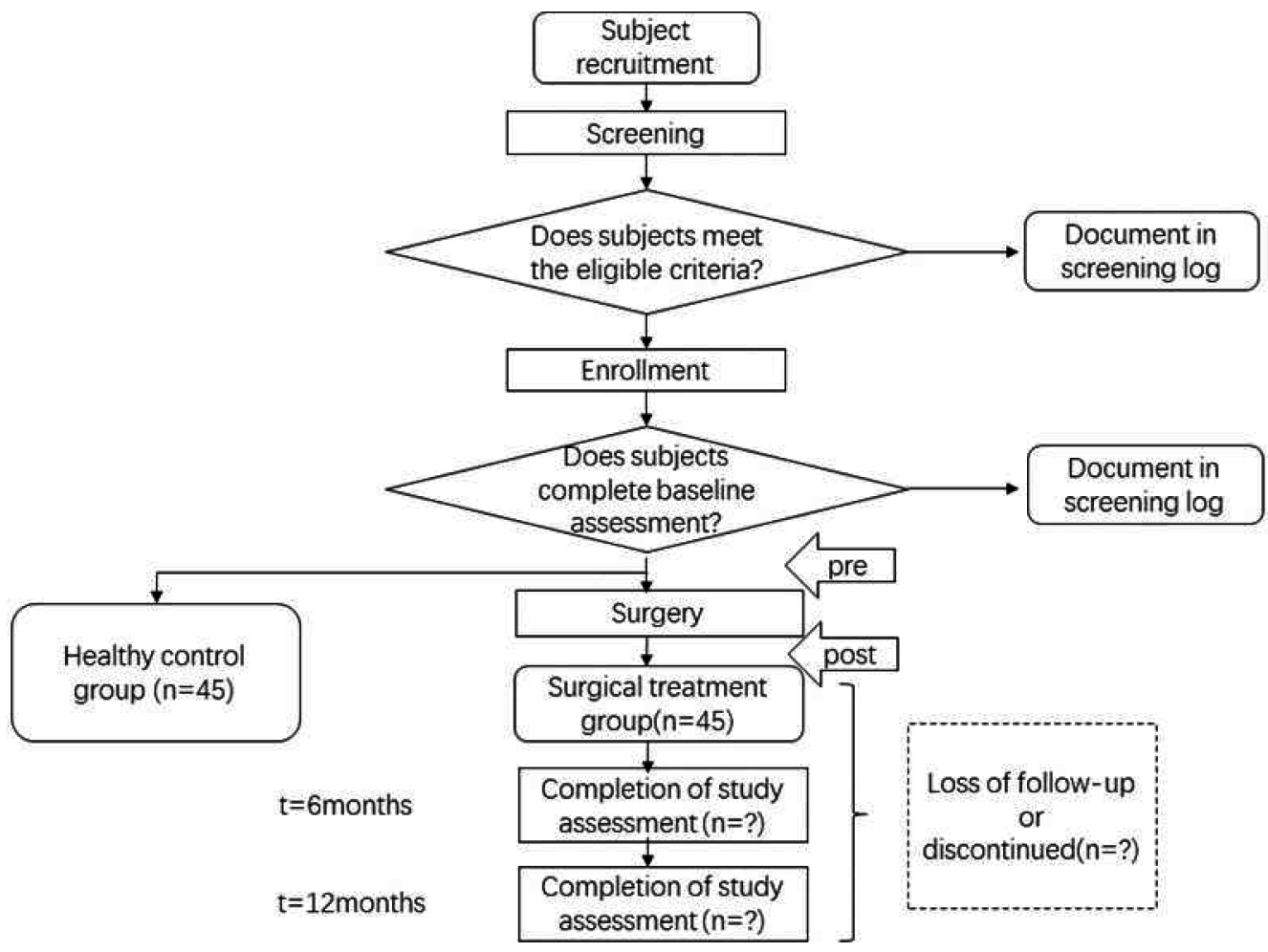

Figure 1 Schematic of the trial design.

the informed consent form. All the identifiable information will be confidential.

Intended subjects will be invited for eligibility and baseline assessments by the research staff. Staff will screen patients who meet the inclusion criteria in the strabismus ophthalmology clinic. As soon as they finish the examinations and meet the inclusion criteria, eligible subjects will be enrolled. The flowchart of the clinical trial is shown in figure 1.

Once the subjects are enrolled, retention efforts will be addressed to participants. The staff of the study will (1) provide periodic contacts about the IXT situation of the subjects, (2) provide feedback regarding the eye conditions and (3) make an appointment for the next review and follow-up visits.

\section{Participants and sample size}

Potential participants will contact study staff and will be provided with an information checklist. Interested participants will complete the clinic screening and, if appropriate, will be invited for an eligibility and baseline assessment by the study staff. The patient will provide written informed consent for participation in the study prior to any trial-specific procedures.

The estimation of sample size will be based on the two following methods: statistical analysis and sample size from previous articles.

\section{Statistical analysis}

The two groups of independent sample rate comparisons will be used for the sample size calculation. According to a previously published article, the improvement rate in distance stereopsis of patients with IXT after surgery was $28 \% .^{29}$ The rate of HCs was supposed to be 0 . Other parameters that were used included $\alpha=0.05,1-\beta=0.8$, twosided and 1:1 allocation to the two study groups. For the above parameters, a $28 \%$ improvement in the ST group versus $0 \%$ in the $\mathrm{HC}$ group was detected at a significance level of $\mathrm{p}<0.05$, and the estimated sample size for each group was 21 subjects. Based on our previous experience with clinical trials for treating IXT, we will estimate the rate of subject loss to follow-up over 1 year to be approximately $50 \%$. Considering these factors together, the estimated sample size for each group will be 42 .

\section{Sample size from previous articles}

Key information about the sample size calculation was missing in published articles related to strabismus, IXT, and fMRI, and a reasonable number of subjects per group ranged from 5 to 32 without follow-up. ${ }^{20} 2830-35$

Based on previous articles and the statistical calculation, a sample of 45 eligible subjects will be required in the ST group of the trial. To compare the changes in the cerebral area, HCs will also be recruited. A total of 45 healthy age-matched HCs who meet the entry criteria will be enrolled.

\section{Eligibility criteria}

Inclusion criteria:

1. Age between 18 and 40 years ${ }^{20} 283133-35$;

2. Evidence of IXT on the basis of history and clinical examination; 
3. Having surgical intention and meeting the surgical indications, including loss of distant stereopsis using synoptophore and/or deflection time accounting for more than half of the waking time; angle of exodeviation $>15^{\wedge}$;

4. No ongoing or planned amblyopia treatment;

5. Best-corrected visual acuity (BCVA) better than or equal to $20 / 20$ in both eyes, no anisometropia $(<1.50$ $\mathrm{D}$ and $<1.00 \mathrm{D}$ in the difference in spherical and cylindrical error, respectively) ${ }^{36}$;

6. Right handedness;

7. Be able to understand and cooperate with examinations and sign informed consent voluntarily.

HCs were recruited based on items of 1, 4, 5, 6 and 7 in the inclusion criteria.

Exclusion criteria (Subjects will be excluded if they meet any of the following criteria):

1. Structural ocular pathology;

2. Previous eye surgery history;

3. Accompanied by vertical strabismus;

4. History of diseases of the central nervous system and the whole body;

5. History of psychiatric diseases, including depressive disorder and delusional disorder;

6. Contraindication for MRI examination (treatable or accidental magnetisable metal in cardiac pacemaker or prosthesis or previous head or spinal trauma requiring neurosurgery, etc.);

7. Claustrophobia.

The study centre is a tertiary A hospital. The ocular examinations of strabismus and the surgery was performed by a chief physician (JF), and a deputy chief radiologist (ZL) performed fMRI examination. Six months after the surgery, patients with the eye deviation less than 10 prism diopters are included. ${ }^{37} 38$

\section{Examinations and study outcomes}

IXT presents as a divergent misalignment of the visual axes. The deviation often becomes manifest with fatigue, visual inattention or illness when fusional compensatory mechanisms are compromised. ${ }^{11}$ Previous studies reported significant changes in the visual cortex in patients with IXT. In addition, the deviation degree, NCS and several influencing factors are also related to the evaluation of IXT. Taken together, in the present project, the primary, secondary and exploratory outcomes are evaluated during the 12-month follow-up period according to the schedule as follows (table 1 and figure 1).

\section{Primary outcome}

Resting-state BOLD-fMRI detection will be performed immediately after recruitment as baseline data and will be evaluated at the 1-year follow-up. For the primary outcome

Table 1 Schedule of assessments and outcomes items

\begin{tabular}{|c|c|c|c|c|c|}
\hline \multicolumn{2}{|c|}{ Procedures/measurements } & \multirow[t]{2}{*}{$\begin{array}{l}\text { Enrolment* } \\
\text { (-2 to } 0 \text { week) }\end{array}$} & \multirow{2}{*}{$\begin{array}{l}\text { Baseline } \\
\times\end{array}$} & \multirow{2}{*}{$\begin{array}{l}6 \text { months } \\
\text { ( } \pm 14 \text { days) } \\
\times\end{array}$} & \multirow{2}{*}{$\begin{array}{l}12 \text { months } \\
\text { ( } \pm 21 \text { days) } \\
\times\end{array}$} \\
\hline Consent form signed & & & & & \\
\hline Basic information & History & & & $x$ & $x$ \\
\hline Optometry examination & Non-cycloplegic autorefraction & & & $x$ & $x$ \\
\hline Visual acuity & Best-corrected visual acuity & & & $x$ & $x$ \\
\hline \multirow[t]{6}{*}{ Strabismus-related tests } & Ocular dominance & & & & \\
\hline & Cover test (distance, near) & & & & \\
\hline & Prism cover test (distance, near) & & & & \\
\hline & Near stereopsis (random-dot stereogram) & & & & \\
\hline & Visual perception examination & & & & \\
\hline & NCS evaluation & & & & \\
\hline \multirow[t]{2}{*}{ Eye examinations } & Slit-lamp exam & & & $x$ & $x$ \\
\hline & Retinal photography & & & $\times$ & $x$ \\
\hline fMRI & Resting-state & $x$ & & $x$ & \\
\hline
\end{tabular}

*Enrolment time-point could be the same day as the baseline measurement.

AC/A, accommodative convergence/accommodation; NCS, Newcastle Control Score. 
analyses, the changes between preoperation and postoperation will be determined by the values between baseline and the last follow-up visit (1 year after the surgery). Other measurements obtained at each follow-up visit will be considered secondary outcome measures.

\section{Procedures for fMRI detection}

After recruitment, an appointment for fMRI examination will be registered by research staff. A resting-state $3.0 \mathrm{~T}$ magnetic resonance scanner (General Electric Medical Systems, Milwaukee, Wisconsin, USA) will be used to scan the visual cortex. Matched eight-channel phased array coil with earplugs and foam padding will be used to lessen scanner noise and head motion. Resting-state fMRI will run for $40 \mathrm{~min}$. Subjects will be asked to close their eyes and stay awake.

\section{Resting-state fMRI}

An EPI pulse sequence will be used, and the imaging protocols will be as follows: a repetition time (TR)/ echo time $(\mathrm{TE})=2000 / 35 \mathrm{~ms}$, flip angle $=90^{\circ}$, field of view $(\mathrm{FOV})=240 \mathrm{~mm} \times 240 \mathrm{~mm}$, matrix $=64 \times 64$. Twentyeight axial slices will be obtained with $4 \mathrm{~mm}$ thickness and a $1 \mathrm{~mm}$ gap. In each fMRI session, the pulse duration will be $400 \mathrm{~s}$, and 3D-BRAVO sequences will be used to acquire high-resolution structural images $(\mathrm{TR}=8.8$ $\mathrm{ms}, \mathrm{TE}=3.5 \mathrm{~ms}$, TI $=450 \mathrm{~ms}$, matrix $=256 \times 256, \mathrm{FOV}=240$ $\mathrm{mm} \times 240 \mathrm{~mm}$, slice thickness $=1.0 \mathrm{~mm}$ without gap, flip angle $=13^{\circ}$ ). During the scan, participants will be required to stay motionless, stay awake, and stay focused until the scan is over.

\section{Secondary outcome}

The following strabismus-related tests are undertaken at each visit (table 1) to assess the basic ocular conditions, the severity of strabismus and the extent of stereopsis defects.

\section{Ocular dominance}

Subjects are asked to hold a card with a central hole and fixate on a distant object while holding their head stationary. The examiner covers the subjects' eyes one after the other and repeats three times to determine the ocular dominance eye. If the results are inconsistent, the dominant eye is recorded as indefinite.

\section{Cover test}

The cover test is used to assess the presence and magnitude of heterophoria and strabismus by asking the subject to fixate on targets at $33 \mathrm{~cm}$ and $6 \mathrm{~m}$, with and without spectacles, if worn, and is conducted by an experienced ophthalmologist. The Hirschberg test is performed to screen for the presence or absence of strabismus. If strabismus is detected, the cover-uncover test is used to differentiate phorias and tropias, to determine if the tropia is intermittent or constant and to differentiate unilateral (right or left) and alternating tropia. If no strabismus is detected, the alternating cover test is performed to detect heterophoria.
Prism cover test

Subjects with strabismus are further measured in prism diopters in the alternating cover test using loose prisms (GZS-01TYPE, Tianyuehengtong Medical Co, Tianjin, China). The prism is placed with the base along the reversed direction of deviation and adjusted until no movement can be detected.

\section{Ocular movements}

To record ocular motility, nine gaze directions, including primary (straight ahead), secondary (right, up, left and down) and tertiary (upper right, lower right, upper left and lower left), are examined and recorded by asking subjects to fix on a moving penlight without moving their heads.

Synoptophore (2001, Clement Clarke, UK) is used to observe the distance stereopsis and accommodative convergence/accommodation (AC/A) ratio. A random-dot stereogram designed by Yan Shaoming and visual perception examination to check the function of near stereopsis is performed. Visual perception examination includes static stereopsis, perceptual eye position and dynamic stereopsis. It is performed on a Windows XP computer mainframe and LG2342p polarised threedimensional displayer. The resolution of the displayer is $1920 \times 1080$, and the refresh frequency is $120 \mathrm{~Hz}$. The visual perception inspection and evaluation system was developed by the National Research Center of Medical and Health Appliance Engineering Technology. During the examination, subjects take the sitting position, with their eyes $0.8 \mathrm{~m}$ away from the centre point of the monitor and at the same height as the monitor. They wear polarised glasses for binocular separation and respond to the examination by mouse or keyboard.

NCS is used to assess how well the squint is controlled by subjects with IXT. ${ }^{39}$ The NCS combines an estimate of the observed frequency of the IXT (home control) with an assessment of the subject's ability to realign the eye following a cover test to induce misalignment (table 2).

\section{Exploratory outcomes}

1. General ophthalmic examinations: slit lamp biomicroscopy (SL-3G, Topcon, Tokyo, Japan) and retinal photography (TRC-50DX/TRC-50DX, Topcon, Tokyo, Japan) are performed to identify any abnormalities of the eye, including anterior segment, refractive media and fundus examination.

2. BCVA: corrected vision at a distance of $6 \mathrm{~m}$ is examined using a LogMAR visual acuity chart.

3. Optometry examination: two drops of $1 \%$ tropicamide were given $5 \mathrm{~min}$ apart to dilate the pupils. Cycloplegic refraction outcome measures will be obtained 30$45 \mathrm{~min}$ after the first drop is instilled, which ensured the maximal cycloplegic effect. Objective refraction is measured before and after cycloplegia using an autorefractor (KR-800, Topcon, Tokyo, Japan), followed by subjective refraction by trained optometrists. 


\begin{tabular}{ll}
\hline Table 2 Newcastle Control Score for IXT (revised) & \\
\hline Control & Score \\
\hline Home & \\
IXT/monocular eye closure seen & \\
Circle appropriate score & 0 \\
Never & 1 \\
$<50 \%$ of time fixing in distance & 2 \\
$>50 \%$ of time fixing in distance & 3 \\
$>50 \%$ of time fixing in distance + seen at near & \\
Clinic control & \\
Circle appropriate score near and distance &
\end{tabular}

Near

\begin{tabular}{ll}
\hline Immediate realignment after dissociation & 0 \\
\hline Realignment with aid of blink or refixation & 1 \\
$\begin{array}{l}\text { Remains manifest after dissociation/prolonged } \\
\text { fixation }\end{array}$ & 2 \\
\hline $\begin{array}{l}\text { Manifest spontaneously } \\
\text { Distance }\end{array}$ & 3 \\
\hline $\begin{array}{l}\text { Immediate realignment after dissociation } \\
\text { Realignment with aid of blink or refixation }\end{array}$ & 0 \\
\hline $\begin{array}{l}\text { Remains manifest after dissociation/prolonged } \\
\text { fixation }\end{array}$ & 2 \\
\hline \begin{tabular}{l} 
Manifest spontaneously \\
\hline
\end{tabular}
\end{tabular}

Total NCS: $($ Home + Near + Distance $)=$

IXT, intermittent exotropia.

\section{Follow-up examinations and measurement schedule}

The examination measures will be assessed at enrolment/ baseline, 6 months and 12 months. The differences in all of the mean values at each follow-up visit from baseline will be analysed (figure 1).

\section{Interventions and allocation}

ST group

Patients with IXT who have a definite diagnosis and conform to surgical indications and agree to ST were included. All of the patients are re-examined according to the schedule (table 1 and figure 1). Before each follow-up visit, the coordinator will make an appointment in advance.

\section{Healthy control $(\mathrm{HC})$ group}

We recruited age-matched, sex-matched and educationmatched volunteers to serve as the HC group. The HC group is set up to determine brain differences between healthy people and patients with IXT.

Ophthalmologists provide advice on the operation strategies according to the subjects' condition and treatment guidelines. The patients freely chose the treatment methods, including whether to have the operation or not. Patients who have both surgical indications and surgical intentions are enrolled in the present study. Therefore, randomisation is not applicable for this trial. Whether the patient chooses to participate in this study will not influence his or her next step in treatment.

\section{Patient and public involvement}

Patients and/or the public were not involved in the design, conduct, reporting, or dissemination plans of our research.

\section{Statistical methods}

Data management and data analyses

For the quantitative data, tests for normality and homogeneity of variance are performed first. Quantitative data conforming to a normal distribution are described as the mean $\pm \mathrm{SD}$. The difference between the ST group and the HC group is compared by a two-sample t test. The difference between the baseline and 12 months after the surgery in the ST group is compared by paired t test. The quantitative data of the skewness distribution are described by the median (Q25, Q75). The Mann-Whitney $\mathrm{U}$ test is used to compare the difference between the ST group and the HC group, and the Wilcoxon signed-rank test is used to compare the difference between the baseline and 12 months after surgery in the ST group. Qualitative data are described as proportions, and the differences between groups are compared by the $\chi^{2}$ test or Fisher's exact test. $\mathrm{P}<0.05$ is considered statistically significant.

\section{General information}

Age from the two groups will be presented as the mean \pm SD. Sex and subject number in each group will be presented as proportions.

\section{Results of fMRI}

The preprocessing was performed using the Data Processing Assistant for Resting-State fMRI (DPARSF 2.1; State Key Laboratory of Cognitive Neuroscience and Learning, Beijing Normal University, Beijing, China; available in the public domain at http://restfmri.net/forum/ DPARSF). It is based on Statistical Parametric Mapping (SPM12) (http://www.fil.ion.ucl.ac.uk/spm/) running by MATLAB R2013b (The MathWorks, Natick, USA). The first 10 volumes will be removed so that participants can adapt to the scanning noise and the signal equilibrium after converting Digital Imaging and Communications in Medicine (DICOM) files to Neuroimaging Informatics Technology Initiative (NIFTIimages. Nex t, we will perform slice timing, head motion correction, spatial normalisation to the Montreal Neurological Institute template (resampling voxel size $=3 \mathrm{~mm} \times 3 \mathrm{~mm} \times 3 \mathrm{~mm}$ ), linear trend removal, temporally bandpass filtering (0.01$0.08 \mathrm{~Hz}$ ) and spatial smoothing with a Gaussian kernel of $4 \mathrm{~mm}$ full-width at half-maximum. If the subject's head shifted by more than $1.5 \mathrm{~mm}$ or rotated by more than $1.5^{\circ}$ during the treatment, the subject's data are discarded. The ALFF and fractional amplitude of low-frequency fluctuation (fALFF) values are calculated using DPARSF. The fALFF is calculated for slow $4(0.027-0.073 \mathrm{~Hz})$ and slow $5(0.01-0.027 \mathrm{~Hz})$ bands. 
Results of ocular examination

Deviation degree, AC/A ratio, near stereopsis, NCS, static stereopsis and perceptual eye position in visual perception examination are described as the mean $\pm \mathrm{SD}$. Far stereopsis and dynamic stereopsis in visual perception examination are described as proportions. Tests for normality and homogeneity of variance are performed first. If the data conform to a normal distribution, we use Pearson correlation analysis to explore the relationships between the mean ALFF/fALFF values of discrepant brain regions and ocular examination in the patient group. Otherwise, we perform Spearman correlation analysis.

\section{Oversight and monitoring}

During the study, data will be entered into a secure database with range checks for each data field. Anonymised paper copies of forms will be stored in secure locked file cabinets. The final dataset will be used after approval by the Steering Committee. Medical records will be kept intact in the hospital. The staff will record the results of the examination on the subject's medical records. The investigator and the ethics committee will be allowed to review the subject's medical records.

Participants can withdraw from the trial without giving a reason. In addition, investigators may also withdraw them from the study to protect participants' safety. Any public report of the results of this study will not disclose the subject's personal identity. We will make efforts to protect the privacy of personal medical data to the extent permitted by law. In accordance with medical research ethics, in addition to personal privacy information, trial data will be available for public inquiry and sharing, which will be limited to web-based electronic databases to ensure that no personal privacy information is disclosed.

We will establish an internal data monitoring committee (DMC) that will consist of ophthalmologists who do not participate in the running trial, statistical experts and ethics committee members. Dr Yan Liu will be the chair of DMC. The DMC will perform data monitoring quarterly, including monitoring safety information, data completeness and adverse events, and so on. The integrity of the trial for each participant will be checked to ensure the appropriate allocation, completeness of outcomes, accuracy, timeliness of data collection, and so on. We will select an independent monitor to check all signed consent forms to ensure validity. All examinations will be general items in daily work and will be non-invasive. The trial will have low risk, and no Data Safety Monitoring Committee will be established for the present trial.

\section{Ethical approval}

The present study was approved by the Medical Ethics Committee of Beijing Tongren Hospital, Capital Medical University (TRECKY2019-147; version number: v1.0; date: 11 December 2019) and conformed to all the principles required by the Declaration of Helsinki.

\section{Potential risks and adverse events}

This is an observational low-risk trial, and risks could come from two aspects. First, the duration of fMRI examination is long, and the average head scan takes approximately 40 min. There is obvious noise in the scanning chamber. Second, MRI is sensitive to the movement of subjects and easily produces artefacts, so the subject cannot move during the scan.

If any discomfort or any unexpected circumstances occur during the study period, whether related to the study or not, we terminate the examination as soon as possible. The participants can withdraw from the study without giving a reason. Doctors do their best to prevent and treat possible injuries from this study. If a subject is injured due to participation in this study, the necessary medical treatment will be provided by the project. According to relevant laws and regulations in China, the research group bears the corresponding medical expenses and provides the corresponding financial compensation.

\section{DISCUSSION}

\section{Binocular vision and IXT occurrence and development}

Binocular fusion includes simultaneous perception, flat fusion and stereopsis according to Worth's classification. ${ }^{40}$ Approximately $70 \%$ of the cells in the striated cortex are binocular cells, and any impairment of fusion and higher spatial perception is closely related to the course of IXT. Fusion function, including sensory fusion and motor fusion, involves the V1, V2, V3, MT regions, and higher-level ventral and dorsal visual pathway coordination. ${ }^{4142}$ Sensory fusion is a cortical process that is defined as the unification of visual excitations from corresponding retinal images into a single binocular stereoscopic visual percept. Motor fusion refers to the ability to fine-tune the eye position to align the eyes and maintain eye alignment, in such a manner that sensory fusion can be maintained. ${ }^{43}$ Any abnormal visual experience, such as anisometropia, ametropia or form deprivation, can destroy binocular fusion by affecting binocular neurons. ${ }^{13}$ The abnormal function of binocular fusion destroys the coordination and balance of the two visual axes. Once the ocular axes separate, visual feedback has no impact on the subsequent outward movement of the deviating eye to its final position, thus leading to eye malposition. After fusion loss, the deviating eye moves outward in a stereotypic, reflexive fashion. ${ }^{45}$ Xue and his colleagues reported that preoperative distance stereopsis and fusion of most patients with IXT were damaged. Six weeks postoperatively, all patients exhibited peripheral fusion, and 98\% demonstrated central fusion compared with $94 \%$ and $40 \%$ preoperatively. The percentage of distance stereoscopic improvement was $77 \%$ compared with $13 \%$ preoperatively. ${ }^{21}$ Strabismus surgery in adults is often regarded as merely cosmetic. However, the ideal goal of surgery is to restore binocular function and stereopsis, as well as motor alignment, ${ }^{37}$ which can improve the healthrelated quality of life in adults. Recently, several studies 
have shown improved sensory status and quality of life in adult patients after successful strabismus surgery. Some adults may also regain fusion and stereopsis and increase the field of binocular vision, suggesting that there may be residual neural plasticity in adulthood. ${ }^{46-49}$

Previous studies have demonstrated the exact visual cortex area of the control centre of fusion and showed changes in the cerebral cortex in patients with exotropia. Yang et al reported that the bilateral frontal gyrus and left lingual visual cortex regulate normal fusion function in human eyes. ${ }^{20} \mathrm{Zhu}$ reported that patients with concomitant exotropia exhibited significantly less functional connectivity (FC) between the left Brodmann area (BA) and left lingual gyrus/cerebellum posterior lobe, right middle occipital gyrus (MOG), left precentral gyrus/ postcentral gyrus and right inferior parietal lobule/ postcentral gyrus and significantly less FC between the right $\mathrm{BA} 17$ and right MOG, which may underlie the pathologic mechanism of impaired fusion and stereopsis in patients with concomitant exotropia. ${ }^{34}$ Shi et al reported that, in patients with constant exotropia, the right V2 showed increased regional homogeneity (ReHo) values, whereas the left BA47 demonstrated decreased spontaneous ReHo values compared with HCs. ${ }^{35} \mathrm{Li}$ et al demonstrated that the fractional anisotropy values in the right inferior frontal-occipital fasciculus (FOF) and right inferior longitudinal fasciculus were significantly higher and that the radial diffusivity values in the bilateral FOF, forceps minor, left anterior corona radiata and left anterior thalamic radiation were significantly lower in the concomitant exotropia group than in the HC group. ${ }^{31}$ With voxel-based morphometry, smaller volumes of gray matter were found in the occipital eye field and parietal eye field, and greater volumes were found in the frontal eye field, supplementary eye field and prefrontal cortex in concomitant exotropia. ${ }^{50}$ Many areas in the cortex and/or subcortical levels of patients with IXT also had abnormal functions. Li et al reported that increased activation intensity was observed in the bilateral MOG, right inferior temporal lobule, right precuneus and fusiform gyrus, and lingual gyrus in the right occipital lobe in subjects with IXT compared with normal subjects. ${ }^{25}$

Overall, assessing the visual cortex of patients with IXT is a way to evaluate changes related to stereopsis and other functions. However, few studies have focused on the plasticity of patients with IXT between cerebral fusion dysfunction and ophthalmic outcomes in follow-ups. In our study, we will perform ophthalmic examinations, such as binocular visual function, NCS and BOLD-fMRI, during the follow-up preoperation and postoperation, which can help us to analyse the changes in fusion function in the eyes and visual cortex comprehensively and provide evidence for the correlation between IXT and cerebral unbalanced activation. Whether the fMRI changes are related to binocular visual function changes or whether there are changes in other cortex will remain unknown until the trial is finished. Perhaps cortical changes will fail to improve binocular visual function, indicating that the changes in the cortex are not synchronised with the alterations of binocular visual function, that the slight changes in the cortex are not enough to induce the changes in binocular visual function or that the existing binocular visual test is not sensitive enough. Even if some regions do not belong to visual cortex, we will also record and analyse them in the present study, because they might also be related to exotropia. The results might provide essential clues to determine potential explanations for the role the central nervous system plays in the pathogenesis and ecology of IXT.

\section{Rationale of the study design}

In recent years, fMRI has become a useful tool for exploring the central mechanism of fusion function as a non-invasive assessment method, which can probably be combined with clinical examination to improve the diagnosis and treatment of IXT. The main advantages of fMRI are high spatial resolution and the ability to reveal detailed microscopic structural changes. ${ }^{22}$ BOLD-fMRI uses the principle of blood oxygenation level dependence, that is, inconsistencies in the local haemodynamics of neurons following excitation, to reveal spontaneous neuronal activity by quantifying alterations in blood oxygen level signals. ${ }^{51}$ In addition, $3.0 \mathrm{~T}$ fMRI has better signal noise and contrast noise than clinically used conventional 1.5 T fMRI. Thus, the present study will employ 3.0 T BOLDfMRI to localise and quantify brain perceptual activities.

Furthermore, resting-state fMRI is an instrumental research method in vision-induced fusion function evaluation. Previously, resting-state fMRI was widely used to evaluate changes in the visual cortex. In our preliminary pre-experimental study, we measured the ALFF values and the fALFF values using resting-state fMRI. Compared with HCs, ALFF/fALFF values significantly increased in the bilateral inferior parietal lobe, MOG and inferior frontal gyrus and decreased in the bilateral postcentral gyrus, precuneus gyrus and right precentral gyrus of patients with IXT. Functional examinations of the visual cortex combined with IXT perioperative evaluation have provided benefits, such as further research about the correlation between visual cortex impairment, plasticity, pathogenesis, treatment and prognosis in patients with IXT .

\section{Potential findings and significance}

In non-human primates, the two eyes are prevented from drifting apart through the regulation of the cortical binocular mechanisms, which maintain fusion. When one eye is occluded, the cortical drive is interrupted, and the covered eye drifts outward. ${ }^{52}$ As shown in our overall study schedule, we will evaluate the changes in the visual cortex before and after the IXT operation and will aim to find the connection between binocular visual function and visual cortex function in patients with IXT. In addition, the findings can provide a non-invasive method for the assessment of visual function via resting-state 
BOLD-fMRI, which may be used as an evaluation method in patients with strabismus and amblyopia.

\section{Trial status}

Participant recruitment will start on 1 September 2021, and the approximate date when recruitment will be completed by is 31 August 2023.

Acknowledgements The authors gratefully acknowledge the financial support of the Beijing Municipal Science \& Technology Commission (Z171100001017066) and High Level Health Technical Talent Training Programme of the Beijing Municipal Health Bureau.

Contributors JF and ZL initiated the study design. YG and JH prepared the consent form. YG, JF and XH drafted and finalised the study protocol. All authors reviewed the study protocol and approved the final manuscript.

Funding The trial is supported by the Beijing Municipal Science \& Technology Commission (Z171100001017066) and the High Level Health Technical Talent Training Programme of the Beijing Municipal Health Bureau (2015-3-023). The funding agencies had no role in the design of the study, data collection, analysis and interpretation of the data, or in writing the manuscript.

Competing interests None declared.

Patient and public involvement Patients and/or the public were not involved in the design, or conduct, or reporting or dissemination plans of this research.

Patient consent for publication Not applicable.

Provenance and peer review Not commissioned; externally peer reviewed. Data availability statement No data are available.

Open access This is an open access article distributed in accordance with the Creative Commons Attribution Non Commercial (CC BY-NC 4.0) license, which permits others to distribute, remix, adapt, build upon this work non-commercially, and license their derivative works on different terms, provided the original work is properly cited, appropriate credit is given, any changes made indicated, and the use is non-commercial. See: http://creativecommons.org/licenses/by-nc/4.0/.

\section{ORCID iD}

Jing Fu http://orcid.org/0000-0002-2939-9479

\section{REFERENCES}

1 Multi-ethnic Pediatric Eye Disease Study Group. Prevalence of amblyopia and strabismus in African American and Hispanic children ages 6 to 72 months the multi-ethnic pediatric eye disease study. Ophthalmology 2008;115:1229-36.

2 McKean-Cowdin R, Cotter SA, Tarczy-Hornoch K, et al. Prevalence of amblyopia or strabismus in asian and non-Hispanic white preschool children: multi-ethnic pediatric eye disease study. Ophthalmology 2013;120:2117-24.

3 Friedman DS, Repka MX, Katz J, et al. Prevalence of amblyopia and strabismus in white and African American children aged 6 through 71 months the Baltimore pediatric eye disease study. Ophthalmology 2009;116:2128-34.

4 Chia A, Dirani M, Chan Y-H, et al. Prevalence of amblyopia and strabismus in young singaporean Chinese children. Invest Ophthalmol Vis Sci 2010;51:3411-7.

5 Matsuo T, Matsuo C. The prevalence of strabismus and amblyopia in Japanese elementary school children. Ophthalmic Epidemiol 2005;12:31-6.

6 Bruce A, Santorelli G. Prevalence and risk factors of strabismus in a UK multi-ethnic birth cohort. Strabismus 2016;24:153-60.

7 Pan C-W, Zhu H, Yu J-J, et al. Epidemiology of intermittent exotropia in preschool children in China. Optom Vis Sci 2016;93:57-62.

$8 \mathrm{Fu}$ J, Li SM, Liu LR, et al. Prevalence of amblyopia and strabismus in a population of 7th-grade junior high school students in central China: the Anyang childhood eye study (ACES). Ophthalmic Epidemiol 2014;21:197-203.

9 Chen X, Fu Z, Yu J, et al. Prevalence of amblyopia and strabismus in Eastern China: results from screening of preschool children aged 36-72 months. Br J Ophthalmol 2016;100:515-9.

10 Zhu H, Yu J-J, Yu R-B, et al. Association between childhood strabismus and refractive error in Chinese preschool children. PLoS One 2015;10:e0120720.
11 Wallace DK, Christiansen SP, Sprunger DT, et al. Esotropia and exotropia preferred practice Pattern $\circledR$. Ophthalmology 2018;125:P143-83.

12 Yao J, Wang X, Ren $\mathrm{H}$, et al. Ultrastructure of medial rectus muscles in patients with intermittent exotropia. Eye 2016;30:146-51.

13 Bui Quoc E, Milleret C. Origins of strabismus and loss of binocular vision. Front Integr Neurosci 2014;8:71.

14 Ahn SJ, Yang HK, Hwang J-M. Binocular visual acuity in intermittent exotropia: role of accommodative convergence. Am J Ophthalmol 2012;154:981-6.

15 Hatt SR, Leske DA, Mohney BG, et al. Fusional convergence in childhood intermittent exotropia. Am J Ophthalmol 2011;152:314-9.

16 Brodsky MC, Jung J, Exotropia I. Intermittent exotropia and accommodative esotropia: distinct disorders or two ends of a spectrum? Ophthalmology 2015;122:1543-6.

17 Hatt SR, Leske DA, Adams WE, et al. Quality of life in intermittent exotropia: child and parent concerns. Arch Ophthalmol 2008;126:1525-9.

18 Wang Y, Xu M, Yu H, et al. Health-related quality of life correlated with the clinical severity of intermittent exotropia in children. Eye 2020;34:400-7.

19 Harwerth RS, Smith EL, Crawford ML. Motor and sensory fusion in monkeys: psychophysical measurements. Eye 1996;10:209-16.

20 Yang X, Zhang J, Lang L, et al. Assessment of cortical dysfunction in infantile esotropia using fMRI. Eur J Ophthalmol 2014;24:409-16.

21 Feng X, Zhang X, Jia Y. Improvement in fusion and stereopsis following surgery for intermittent exotropia. J Pediatr Ophthalmol Strabismus 2015;52:52-7.

22 Miki A, Haselgrove JC, Liu GT. Functional magnetic resonance imaging and its clinical utility in patients with visual disturbances. Surv Ophthalmol 2002;47:562-79.

23 Chen X, Zirnsak M, Vega GM, et al. Parietal cortex regulates visual salience and Salience-Driven behavior. Neuron 2020;106:177-87.

24 Jamadar SD, Ward PG, Li S, et al. Simultaneous task-based BOLD-fMRI and [18-F] FDG functional PET for measurement of neuronal metabolism in the human visual cortex. Neuroimage 2019;189:258-66.

25 Li Q, Bai J, Zhang J, et al. Assessment of cortical dysfunction in patients with intermittent exotropia: an fMRI study. PLoS One 2016;11:e0160806.

26 Yan X, Lin X, Wang Q, et al. Dorsal visual pathway changes in patients with comitant extropia. PLoS One 2010;5:e10931.

27 Huang X, Li S-H, Zhou F-Q, et al. Altered intrinsic regional brain spontaneous activity in patients with comitant strabismus: a resting-state functional MRI study. Neuropsychiatr Dis Treat 2016;12:2949-56.

28 Tan G, Huang X, Zhang Y, et al. A functional MRI study of altered spontaneous brain activity pattern in patients with congenital comitant strabismus using amplitude of low-frequency fluctuation. Neuropsychiatr Dis Treat 2016;12:1243-50.

29 Morrison D, McSwain W, Donahue S. Comparison of sensory outcomes in patients with monofixation versus bifoveal fusion after surgery for intermittent exotropia. J Aapos 2010;14:47-51.

30 Tan G, Dan Z-R, Zhang Y, et al. Altered brain network centrality in patients with adult comitant exotropia strabismus: a resting-state fMRI study. J Int Med Res 2018;46:392-402.

31 Li D, Li S, Zeng X. Analysis of alterations in white matter integrity of adult patients with comitant exotropia. J Int Med Res 2018;46:1963-72.

32 Yan X, Wang Y, Xu L, et al. Altered functional connectivity of the primary visual cortex in adult comitant strabismus: a resting-state functional MRI study. Curr Eye Res 2019;44:316-23.

33 Ouyang J, Yang L, Huang $X$, et al. The atrophy of white and gray matter volume in patients with comitant strabismus: evidence from a voxel-based morphometry study. Mol Med Rep 2017;16:3276-82.

34 Zhu P-W, Huang X, Ye L, et al. Altered intrinsic functional connectivity of the primary visual cortex in youth patients with comitant exotropia: a resting state fMRI study. Int $J$ Ophthalmol 2018;11:668-73.

35 Shi $\mathrm{H}$, Wang $\mathrm{Y}$, Liu $\mathrm{X}$, et al. Cortical alterations by the abnormal visua experience beyond the critical period: a resting-state fMRI study on constant exotropia. Curr Eye Res 2019;44:1386-92.

36 Margines JB, Huang C, Young A, et al. Refractive errors and amblyopia among children screened by the UCLA preschool vision program in Los Angeles County. Am J Ophthalmol 2020;210:78-85.

37 Mills MD, Coats DK, Donahue SP, et al. Strabismus surgery for adults: a report by the American Academy of ophthalmology. Ophthalmology 2004;111:1255-62.

38 Carruthers JD, Kennedy RA, Bagaric D. Botulinum vs adjustable suture surgery in the treatment of horizontal misalignment in adult patients lacking fusion. Arch Ophthalmol 1990;108:1432-5. 
39 Buck D, Clarke MP, Haggerty H, et al. Grading the severity of intermittent distance exotropia: the revised Newcastle control score. Br J Ophthalmol 2008;92:577.

40 Squint: its causes, pathology and treatment. Edinburgh Medical Journal 1903;14:350-50 https://www.ncbi.nlm.nih.gov/labs/pmc/ articles/PMC5164621/

41 Cox MA, Dougherty K, Westerberg JA, et al. Temporal dynamics of binocular integration in primary visual cortex. J Vis 2019;19:13.

42 Grossberg S, Srinivasan K, Yazdanbakhsh A. Binocular fusion and invariant category learning due to predictive remapping during scanning of a depthful scene with eye movements. Front Psychol 2014;5:1457.

43 O'Connor AR, Birch EE, Anderson S, et al. Relationship between binocular vision, visual acuity, and fine motor skills. Optom Vis Sci 2010;87:942-7.

44 Fredenburg P, Harwerth RS. The relative sensitivities of sensory and motor fusion to small binocular disparities. Vision Res 2001;41:1969-79.

45 Economides JR, Adams DL, Horton JC. Capturing the moment of fusion loss in intermittent exotropia. Ophthalmology 2017;124:496-504.
46 Dickmann A, Aliberti S, Rebecchi MT, et al. Improved sensory status and quality-of-life measures in adult patients after strabismus surgery. J Aapos 2013;17:25-8.

47 Mets MB, Beauchamp C, Haldi BA. Binocularity following surgical correction of strabismus in adults. J Aapos 2004;8:435-8.

48 Morris RJ, Scott WE, Dickey CF. Fusion after surgical alignment of longstanding strabismus in adults. Ophthalmology 1993;100:135-8.

49 Koc F, Erten Y, Yurdakul NS. Does restoration of binocular vision make any difference in the quality of life in adult strabismus. $\mathrm{Br} \mathrm{J}$ Ophthalmol 2013;97:1425-30.

50 Chan S-T, Tang K-W, Lam K-C, et al. Neuroanatomy of adult strabismus: a voxel-based morphometric analysis of magnetic resonance structural scans. Neuroimage 2004;22:986-94.

51 Shao Y, Li Q-H, Li B, et al. Altered brain activity in patients with strabismus and amblyopia detected by analysis of regional homogeneity: A resting-state functional magnetic resonance imaging study. Mol Med Rep 2019;19:4832-40.

52 Coppola D, Purves D. The extraordinarily rapid disappearance of entoptic images. Proc Natl Acad Sci U S A 1996;93:8001-4. 